\title{
The Interplay between Individual and Collective Activity: an Analysis of Classroom Discussions about the Sierpinski Triangle
}

\author{
Geoffrey B. Saxe ${ }^{1}$ (D) Amelia M. Farid ${ }^{1}$
}

Accepted: 11 July 2021/Published online: 06 October 2021

(C) The Author(s) 2021

\begin{abstract}
This article uses a cultural-developmental framework to illuminate the interplay between collective and individual activity in the mathematical reasoning displayed in a university Masters level lesson on fractals. During whole class and small group discussions, eleven students, guided by an instructor, engage in inductive reasoning about the area and perimeter of the Sierpinski triangle, a unique mathematical object with zero area and infinite perimeter. As participants conceptualize and communicate about the Sierpinski problem, they unwittingly generate a linguistic register of action word forms (e.g., fencing, zooming) and object word forms (e.g., area, infinity) to serve Sierpinski-linked mathematical reasoning functions, a register that we document in the first analytic section of the article. In the second analytic section, we report a developmental analysis of microgenetic, ontogenetic, and sociogenetic shifts in the word forms constitutive of the register and their varied functions in participants' activities. In the third analytic section, we provide a cultural analysis of the classroom's collective practices, practices that enable and constrain participants' constructions of formfunction relations constituting the register. We examine the ways in which participants work to establish a common ground of talk in their communicative exchanges, exchanges supported by classroom norms for public displays of reasoning and active listening to one another's ideas. We show that it is as participants work to establish a common ground that the register emerges and is reproduced and altered. We conclude by pointing to ways that the analytic framework can be extended to illuminate learning processes in other classroom settings.
\end{abstract}

Keywords Classroom interaction - Mathematics education - Linguistic register · Inductive reasoning $\cdot$ Language-thought relations

Geoffrey B. Saxe

saxe@berkeley.edu

1 University of California, Berkeley, Berkeley, CA, USA 
In this article, we use a cultural-developmental framework (Saxe, 2012, 2015; Saxe et al., 2015a, b) to guide our analysis of the interplay between collective and individual activity in emergent mathematical reasoning during a university lesson involving the Sierpinski triangle, a baffling mathematical object with infinite perimeter and zero area. We argue that to understand the interplay requires two intertwined analytic strands: developmental analyses of individuals' use of representational forms to serve reasoning and communicative functions as they participate in the collective practices of classroom life, and cultural analyses of how individuals' participations often unwittingly reproduce and alter collective practices, including emergent participation structures, social positions, norms, and linguistic registers.

In our analysis, we focus on an emerging linguistic register, a set of word forms that comes to be used to serve specialized functions among members of a community. We show that classroom participants' sustained and engaged interaction about the baffling problem was fertile ground for the emergence of the novel register. Indeed, we reveal how participants' bafflement led them to generate, communicate about, struggle with, and work through novel ideas, unwittingly using word forms to serve new functions. For example, to think through and communicate about the curious object of a perimeter with infinite length, a participant in the Sierpinski classroom recruited the word form fencing to represent perimeter, a novel function for fencing that became used and reused in the community to support reasoning and communication.

The focus on an emerging linguistic register in a university classroom community constitutes a novel application of our cultural-developmental approach. In prior work, the cultural-developmental approach was limited to mathematics in preschool (e.g., Saxe et al., 1987), elementary school (e.g., Saxe, de Kirby, Kang, et al., 2015a; Saxe, de Kirby, Le, et al., 2015b), and secondary school (e.g., Saxe et al., 2009) as well as elementary mathematics in out-of-school practices, like candy selling in urban Brazil (e.g., Saxe, 1991), straw weaving in rural Brazil (e.g., Saxe \& Gearhart, 1990), and economic exchange in remote Papua New Guinea communities (e.g., Saxe, 2012). We now extend the approach to address issues in tertiary mathematics. Further, although in the Papua New Guinea case, a novel linguistic register was included as a focus of the cultural-developmental approach, the empirical analyses were limited to understanding form-function shifts over historical time, not the dynamics of face-to-face interactions in which a register might emerge (e.g., Saxe, 2012). In the present Sierpinski study, we conduct microethnographic analyses of face-to-face interactions with a focus on an emerging register. Finally, to reveal interactional dynamics leading to the emergence of the register in the collective practices of the Sierpinski classroom, we make use of novel methodological techniques, techniques that may be useful to others engaged with in situ analyses of reasoning and novel uses of language in the collective practices of a community.

The context for our analysis is a lesson in a university Masters level course for preservice teachers titled, "Chaos and Fractals for Masters in Mathematics Education." Our analyses focus on one part of the lesson, which we refer to as the Conjectures Discussions. The opportunity to undertake the analysis came about through a small workshop in which organizers invited several researchers to analyze the same segment of video data. The video data afforded us the opportunity to illustrate some key aspects of our cultural-developmental analytic approach and its potential utility in analyzing classroom worlds. 
The classroom participants in the video recording included 11 students, nine of whom were active in the Conjectures Discussions featured in our analysis, and two coinstructors, one of whom (referred to in this article as "the instructor") was active in the Conjectures Discussions. During the Conjectures Discussions, participants reflect on and communicate with one another about the self-similar iterative constructions of the Sierpinski triangle (depicted in Fig. 1), constructions in which, as the area decreases over iterations, the cumulative perimeter increases. Formally, evaluating the area and perimeter of the completed Sierpinski triangle requires mathematical induction: an analysis of (i) a base stage - the structure of one iteration in the Sierpinski transformation, (ii) successive stages - the consequences of each recursive self-similar transformation for the triangle's area and perimeter, and (iii) an end state-the hypothetical conclusion of infinite iterative transformations.

\section{The Context for our Analysis: The Sierpinski Triangle Lesson}

Throughout the lesson, the instructors orchestrated two kinds of classroom organizations-whole class discussions and small group discussions with 2 or 3 students per group. Figure 2 contains a representation of lesson segments that were preparatory for the Conjectures Discussions and the lesson segments constituting the Conjectures Discussions.

The preparatory lesson segments depicted in Fig. 2 provided context for the Conjectures Discussions. In the first whole class preparatory segment, the coinstructors presented a worksheet to the small groups along with erasable felt-tipped markers and large white boards. The worksheet included procedures for the Sierpinski geometrical construction (see Fig. 1). Once groups constructed several Sierpinski iterations over a 20 min interval, they engaged in a preliminary whole class discussion about their constructions (see Fig. 3). One of the instructors then asked the small groups to make a conjecture about area of the Sierpinski triangle, initiating inductive reasoning about area and perimeter.

The Conjectures Discussions consisted of a whole class discussion, a groupwork segment (recorded in two of the four small groups), and a second whole class discussion, during which the class grappled with the questions of area and perimeter.

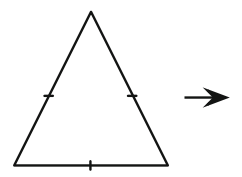

a. equilateral triangle

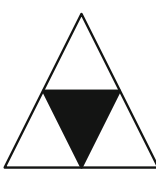

b. first iteration

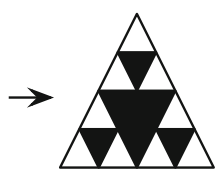

c. second iteration

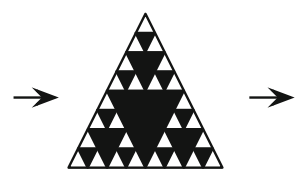

d. third iteration

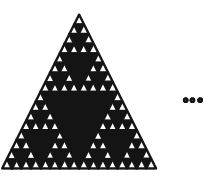

e. fourth iteration

Fig. 1 Iterative self-similar constructions of the Sierpinski triangle. (a) Midpoints for each side of an equilateral triangle are identified and then (b) connected, resulting in a to-be-removed inverted equilateral triangle (shaded) with three upright equilateral triangles that are not removed (unshaded). Subsequently, (c,d,e) self-similar constructions are repeated infinitely within each remaining unshaded upright equilateral triangle ( $2 \mathrm{nd}, 3 \mathrm{rd}, 4 \mathrm{th}, \ldots$ iterations). As progressively more shaded triangles are removed from the construction, the area of the fractal decreases and the cumulative perimeter of the fractal increases. Infinitely many such iterations result in a (counterintuitive) mathematical construct with zero area and infinite perimeter 


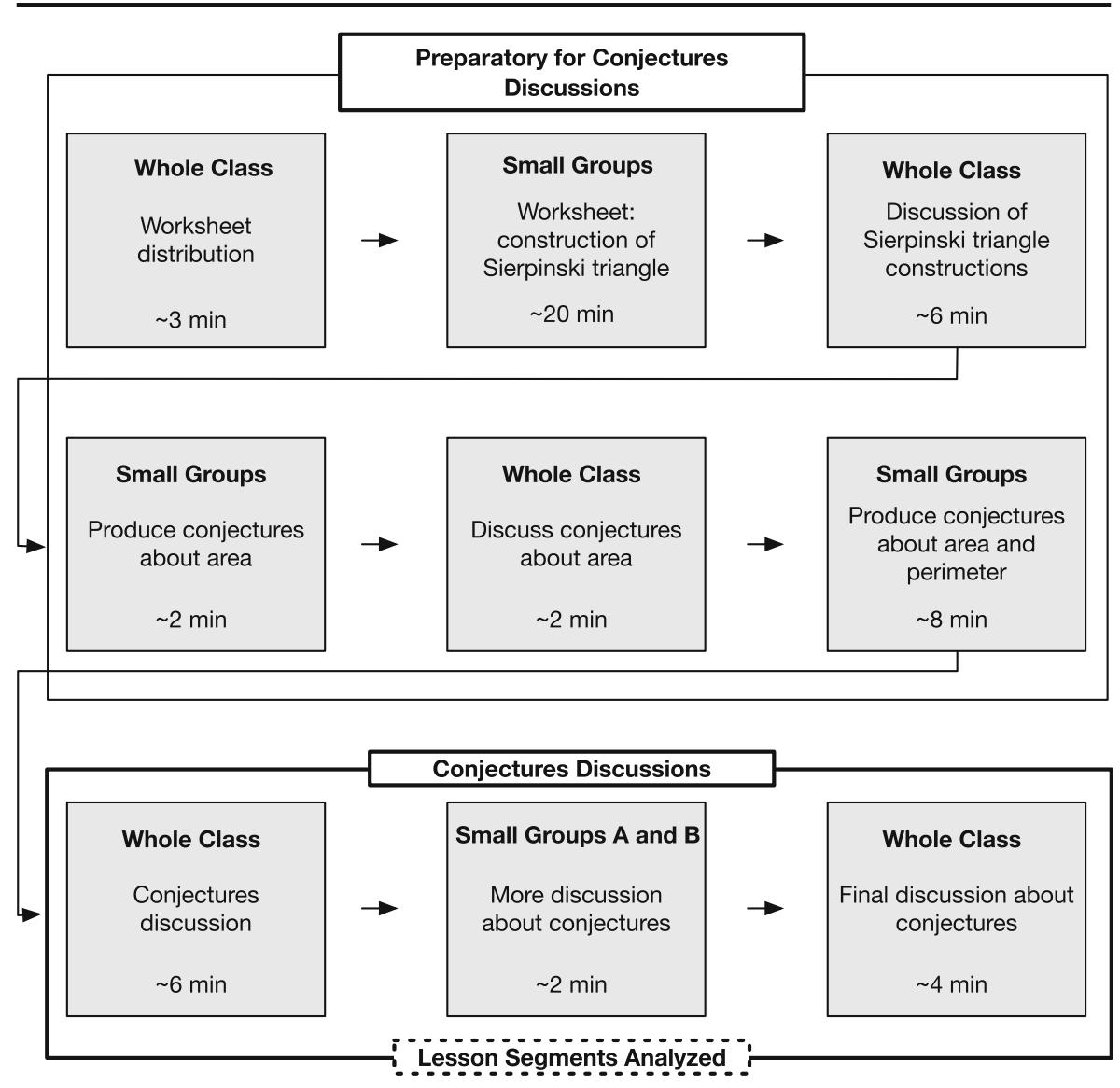

Fig. 2 Situating the Conjectures Discussions in the organization of the lesson

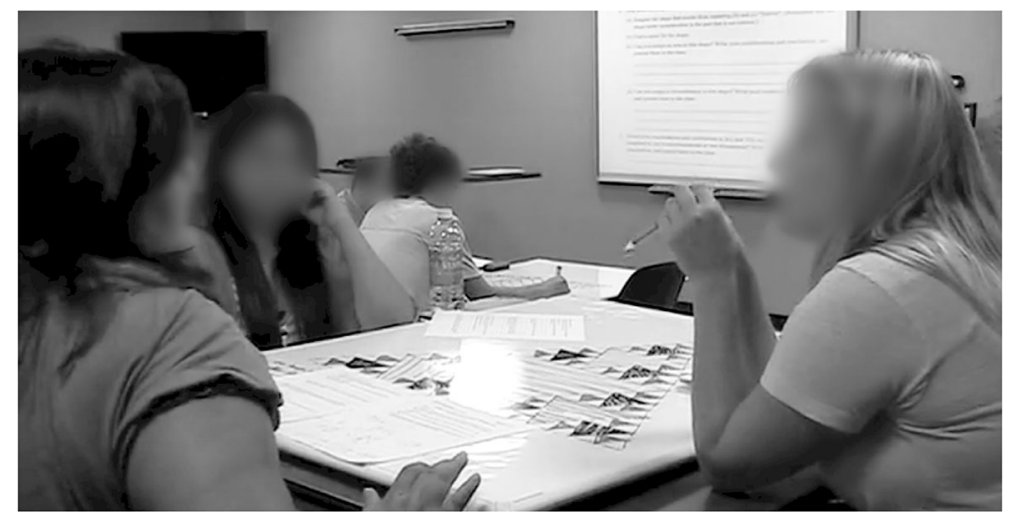

Fig. 3 Small Group A working on the material construction of the Sierpinski Triangle prior to the Conjectures Discussions 


\section{Introducing the Framework: Carmen's Conjecture}

We introduce our cultural-developmental framework with an example of a student's remark early in the Conjectures Discussions, a remark that we will argue constitutes a moment in the emergence of a linguistic register in the classroom community. In response to an instructor's request to explain her thinking about the Sierpinski triangle, the student, Carmen, conjectures that the area and perimeter of the triangle both resolve to zero.

Carmen: Okay, for our area thing, we were going to keep zooming in, keep coloring in, so eventually we're gonna color all in. It's going to be black, so there's no area, so there's nothing to... No area there's nothing to put a fence around it. So, there'd be no perimeter...

We regard Carmen's statement as cryptic and complex. Carmen uses word forms for geometrical objects, like perimeter and area; these word forms take on multiple referential functions as we will show in our analysis. She also refers to actions not referenced in the problem, using word forms like zooming, coloring and put a fence. In fact, all of these word forms are polysemous and could be interpreted in any number of ways by other classroom participants. Zooming, for example, could refer to going fast. Fencing could refer to a sword fight competition. Coloring could refer to what a child does in drawing with crayons. Area could refer to part of a town. But Carmen uses these words in specialized ways, tailoring them to conceptualize and address the Sierpinski problem.

In our analyses, we leverage our cultural-developmental framework to show how a linguistic register emerges and comes to be reproduced and altered in the collective practices of Carmen's classroom community, a linguistic register that includes Carmen's use of both action word forms, like zooming or fencing, and object word forms, like specialized uses of area or perimeter. Further, we argue that the register comes to figure centrally in participants' conceptualizing activity as well as participants' developments of mathematical ideas.

In this section we introduce first the developmental and then the cultural aspects of our analytic framework. ${ }^{1}$ We then turn to our analyses of the Conjectures Discussions.

\section{Developmental Aspects of the Framework: A Focus on Form-Function Relations and an Emerging Linguistic Register}

Cognitive development is constituted through microgenetic, sociogenetic, ontogenetic, as well as phylogenetic processes (Cole \& Hatano, 2007; Saxe, 2012; Saxe \& Esmonde, 2005). In our approach, we focus on individuals' use of speech forms, like Carmen's use of zooming, perimeter, or fencing, to serve cognitive and communicative functions with a concern for individuals' generation of form-function relations in microgenetic, sociogenetic, and ontogenetic processes.

\footnotetext{
${ }^{1}$ We find points of convergence in our treatment of form-function relations in communication with a number of authors, including Anna Sfard's treatment of thinking as communicating (Sfard, 2008) and Dan Slobin's treatment of thinking for speaking (Slobin, 1996, 2003).
} 


\section{Microgenesis: Schematizing Form-Function Relations}

The microgenesis of form-function relations is a developmental process that occurs over a short duration of time. Various scholars have addressed the import of microgenetic analyses in cognitive development, pointing to the agentive role of the individual in generating knowledge and in problem solving. In their seminal writings, Vygotsky's concern with mediated activity (Vygotsky, 1978, 1986) and Piaget's focus on individuals' constructive activities in their solutions to cognitive tasks (Piaget, 1970a, 1970b) are examples of such scholarly commitments. In our treatment, we find particularly apt aspects of Werner and Kaplan's approach to microgenesis, specifically with regard to their treatment of symbol formation (Werner \& Kaplan, 1963; see also Langer, 1970).

In their treatment, Werner and Kaplan elaborate an approach to microgenesis as a process of "dynamic schematization." In their use of this term, Werner and Kaplan meant that individuals do not passively internalize symbols or meanings for symbols. Neither do they simply associate symbols with preformed meanings. In their treatment, Werner and Kaplan argue that the microgenetic process is constituted as individuals structure, in concert and over a short duration of time: (a) a symbolic vehicle, like the word forms that Carmen deploys, (b) referential objects, like Carmen's conceptualizations of the effects of Sierpinski transformations, and (c) vehicle-object relations as the vehicle and object come to reciprocally inform one another in the microgenesis of the representational act.

To illustrate how we will build upon Werner and Kaplan's account of microgenesis, let us consider again Carmen's statement, "we were going to keep zooming in, keep coloring in," as it relates to Sierpinski transformations. As we depict in Fig. 4, Carmen's use of zooming and coloring (a. symbolic vehicle) and her conceptualization of Sierpinski transformations (b. referential object) become dynamically related in her schematizing activity, with the word forms and her conceptualizations informing one another in the microgenetic process (c. vehicle-object relations). In generating vehicleobject relations, Carmen's effort to conceptualize the object - the successive Sierpinski stages - serves as a context for her to recruit a vehicle, zooming and coloring. Reciprocally, in the use of particular a vehicle, zooming and coloring, Carmen crystallizes her referential object, Sierpinski transformations.

(a) Symbolic Vehicle

zooming, coloring

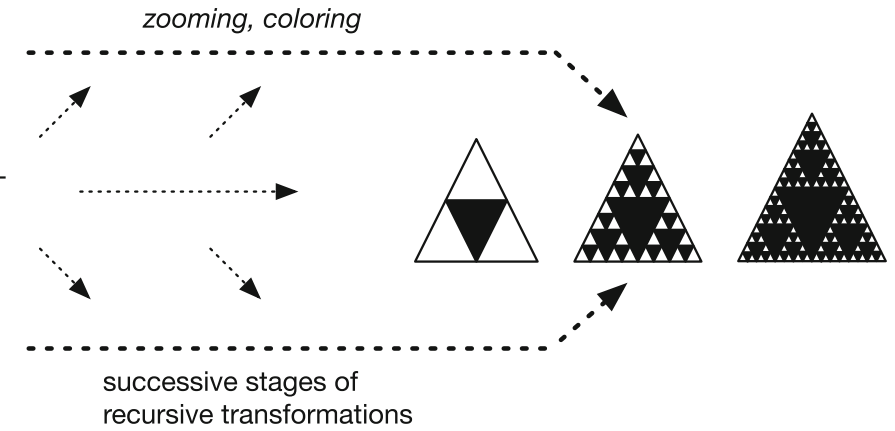

Fig. 4 A process of dynamic schematizing in her microgenesis of zooming and coloring: Carmen's coextensive generation of a (a) symbolic vehicle, (b) referential object, and (c) vehicle-object relations 


\section{Sociogenesis: Reproducing and Altering Form-Function Relations}

Number words and notational systems, mathematical conjectures and theorems, and the myriad word forms that populate human languages are all sociogenetic developments. In human activity, these varied forms have been reproduced and altered over the complex interweaving of social histories of communities. On a much smaller scale and over a short time period in the Conjectures Discussions, we regard an emerging linguistic register used to serve Sierpinski-linked functions as a sociogenetic development.

As word forms and the functions they serve become reproduced by others in a sociogenetic process, they become detached from the actions of any one individual (cf. Croft, 2000; Keller, 1994). Consider Carmen's use of the linguistic form fence, followed immediately by the instructor, who states, "there's nothing left to put a fence around..." and subsequently by Jen, who states “... if you zoom in there's more to fence ..." Each individual takes up the word form fence to serve functions related to mathematical induction in considering the perimeter of the Sierpinski Triangle, reproducing the form-function relations introduced by Carmen. As they do so, fencing emerges as constitutive of a linguistic register in the Sierpinski classroom, detached from its use by any one individual. The sociogenetic dynamics whereby the linguistic register emerges and is reproduced and altered are a focus in our developmental analyses of the Conjectures Discussions.

\section{Ontogenesis: Generating Continuities and Discontinuities in Development}

The study of the ontogeny of cognition - cognitive development over the lifespan of individuals - has long been a focus in developmental psychology, whether Piaget's treatment of cognitive structures (Piaget, 1970b), Vygotsky's focus on mediation (Vygotsky, 1978), or Werner's focus on the orthogenetic principle (Werner, 1948). In these treatments and subsequent work, individuals' cognitive developments are studied over substantial time periods and often captured through cross-sectional quasi-experimental designs using interview techniques. In the case of our analyses presented here, we have adopted a more limited approach due to constraints on the data corpus noted earlier.

In cognitive developmental treatments of ontogenesis, a fundamental concern is how individuals maintain the integrity of thought in the context of change (see, for example, diSessa, 2017; Langer, 1970; Piaget, 1970b; Vygotsky, 1986; Werner, 1948). How does Carmen maintain the integrity of her thought as she adapts her thinking and her communication over the course of the lesson? We address this question with an analysis of continuities and discontinuities in relations between word forms and their functions. Carmen draws on word forms like fence that presumably served functions unrelated to mathematical induction in her own prior everyday talk and actions. In her use of a form that she has previously used, she shows a continuity in use of word forms. At the same time, Carmen extends fence to serve novel functions related to mathematical induction in the Sierpinski case, and thus her particular use of fence illustrates a discontinuity in function, a function that is specific to the emerging linguistic register in the Conjectures Discussions. 


\section{Cultural Aspects of the Framework: A Focus on Collective Practices and the Perpetual Reconstruction of a Linguistic Register in Talk}

The approach we take in the second strand of our analysis is cultural in a sense different from the one often used in everyday talk and in some scholarly treatments. "Cultures" are often treated as bounded, homogeneous, and coherent, as in "French culture" or "classroom cultures." But like others who problematize the word (Sperber \& Hirschfeld, 1999), we take there to be no "cultures." Rather, we find only cultural phenomena: the reproduction and alteration of norms, conventions, social positions, roles, and, more generally, a common ground of talk and action that is inherently in flux, generated and re-generated in activity (Saxe \& Esmonde, 2005). The common ground of talk and action includes a linguistic register, the focus of this article. In our analyses of the interactions in the Sierpinski classroom and the emergence of a linguistic register, we consider the production of cultural phenomena and their intrinsic relation to developmental processes.

\section{Collective Practices as Microcosms}

The construct of "practice" or "cultural practice" has a history of use in cultural treatments of cognitive development. "Practice" often refers to a recurrent activity with which an individual engages linked to a cultural group. A child counting with English number words, an adult commuting to work, and a Papua New Guinean woman engaged with cultural-specific slash and burn gardening techniques are all examples of "cultural practices." Our aim is to capture not simply recurrent actions of individuals, but rather the recurring organizations of joint or collective activities, or "collective practices." In collective practices, participants' actions, as they interact with one another, take form in relation to participation norms (Yackel \& Cobb, 1996), social positions/roles (Harré, 2012; Hirvonen, 2016), and conventions (Lewis, 1969; Saxe, 1991). With a focus on the interplay between individual and joint activity, we treat collective practices as microcosms that can illuminate the production and reproduction of cultural phenomena in which developmental processes are situated.

The whole class discussion in which Carmen participates is an example of a collective practice. When Carmen responds to the instructor's request, the instructor, taking up an expected social position of authority and the role of leader, asks Carmen for a display of her thinking; in turn, taking up the position of student, Carmen responds, abiding by a norm in the collective practice that students should display their thinking publicly. As we will show, other participants in the discussion also conform to this norm and share their thinking publicly, and, in so doing, schematize word forms like zooming and fence. Participants' microgenetic productions become constitutive of the collective practice, both through their generation of specialized form-function relations of the register and through their reproduction of the norm to display problem-related thinking publicly.

\section{Common Ground and Collective Practices}

Our analyses include a focus on a common ground of talk in the Conjectures Discussions, a construct that illuminates the emergence, reproduction, and 
alteration of the linguistic register. By "common ground," sociolinguistic scholars generally mean shared knowledge of word meanings and norms for communication, shared knowledge that enables successful communication and coordinated action (cf. Allan, 2013; Clark, 1985; Stalnaker, 2002). We take the generation of a common ground to involve the active involvement of speakers and listeners and to be inherently problematic for interlocutors. In conversation, listeners have no direct access to speakers' intended meanings, and speakers have no direct access to the knowledge and interpretive frames that listeners bring to communicative interactions. As a consequence, interlocuters must read through one another's communicative displays in order to interpret each other's meanings, and to do so, interlocuters need to "cooperate" (Grice, 1989). Speakers typically presuppose what knowledge listeners bring to the interaction and frame their utterances so their listeners will understand their intended meanings; in concert, listeners typically presuppose that speakers are trying to support understanding of their intended meanings, not speaking falsely or attempting to misguide.

In the excerpt we have been discussing, Carmen likely presupposes that her listeners will extend their everyday meanings of her words zooming, fencing, and perimeter to functions linked to the Sierpinski problem. And Carmen's listeners likely do their best to make sense of Carmen's new functions for these word forms in relation to the Sierpinski problem. In our treatment of collective practices, we take common ground to be perpetually generated and re-generated in interaction, and we also assume that the relation between participants' utterances and their thinking is perpetually problematic, since one's utterances can never capture the entirety of one's thinking. Common ground is at best 'taken-as-shared' by interlocutors, since interlocutors can never know one another's minds directly (Blumer, 1969; Yackel \& Cobb, 1996). Our conceptualization of common ground is central to understanding the emergence, reproduction, and alteration of a linguistic register in the collective practices of the Sierpinski classroom.

\section{Cultural and Developmental Processes As Intrinsically Related}

We argue that our analysis of the linguistic register in the Conjectures Discussion provides an illustrative case of intrinsic relations between cultural and developmental processes. Figure 5, adapted from Saxe (2012), contains a schematic of the instructor and two students, Carmen and Joy, with their ontogenetic developments represented by horizontal parallel lines moving in time from past to present to future. These participants' ontogenetic developments become constituted through their own microgenetic schematizations of form-function relations within the collective practices of the classroom. These schematizations are enabled and constrained, on the one hand, by the cognitive resources that individuals have generated in their prior microgenetic acts, and, on the other, in their incorporation of sociogenetic developments of forms and functions, such as the emerging linguistic register that has been reproduced and altered over the Conjectures Discussions as participants work to establish a common ground as they engage in communication and problem solving with one another. 


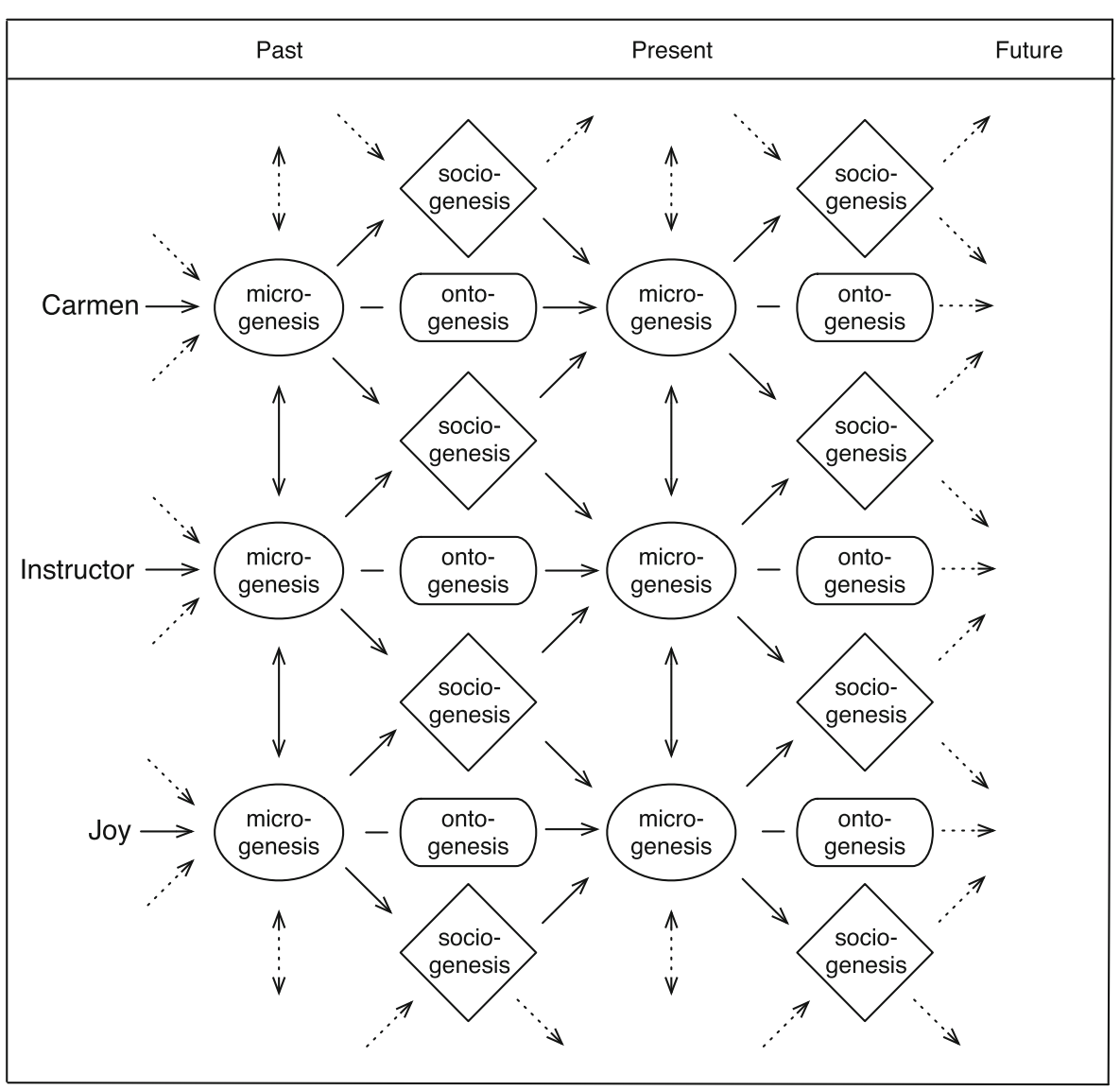

Fig. 5 The interplay between microgenetic, ontogenetic, and sociogenetic processes over time within the collective practices of the Sierpinski classroom

\section{Analyzing the Conjectures Discussions}

Figure 6 provides an overview of our three-part approach to analysis. In the first part, we document the use of action and object word forms in the classroom community, word forms that we argue become constitutive of a linguistic register. In the second part, we analyze evidence of cognitive development as participants elaborate word

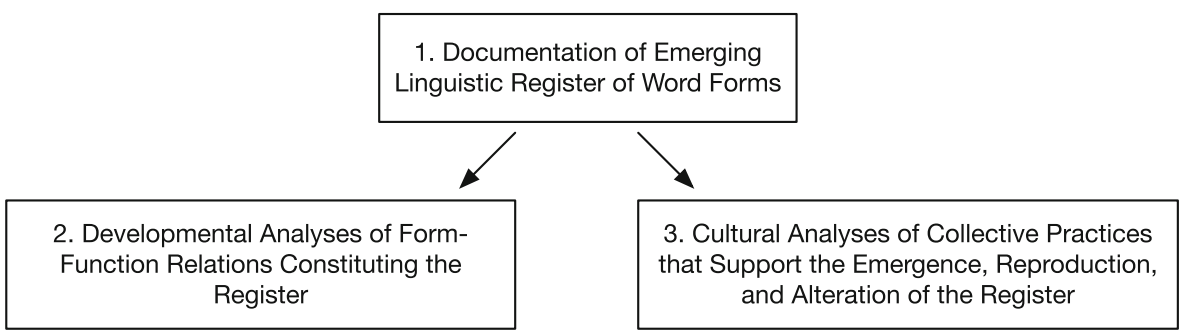

Fig. 6 The three parts of our analysis 
form-function relations in processes of microgenesis, sociogenesis, and ontogenesis. In the third part, we focus on cultural processes, the collective practices in which participants' elaboration of form-function relations are situated.

\section{Documentation of an Emerging Linguistic Register of Word Forms}

We identified 10 word forms as candidates for the emerging linguistic register. These included word forms representing actions (such as coloring) and word forms representing objects that are acted on (such as area). We differentiate between action and object forms due to differences in the functions that they served as participants conceptualized the problem of the Sierpinski Triangle, differences in functions that we will address shortly. The action word forms we analyzed include coloring, shading, inking, fencing, zooming, and converging. The object word forms include both geometrical objects, area and perimeter, and numerical objects, zero and infinity. The bases for selection of word forms as candidates for inclusion were that (a) each word form appeared to be used to accomplish functions linked to the Sierpinski problem of induction and that (b) any candidate word form had to be used by at least two participants. The list of included word forms was not intended to be exhaustive, but only to serve as illustrative exemplars. We anticipated variation in use of these word forms, because varying longevities of word forms and intensity of use over time is to be expected in processes of the reproduction and alteration of a linguistic register.

Figure 7 contains timeline representations for the key word forms that became constitutive of the emerging linguistic register. Included are each form's frequency of occurrence $(n)$ and when each form was used (represented by hatch marks). Use of a single form occurred multiple times in close temporal proximity is represented as a single hatch mark of greater breadth. The temporal duration, in minutes and seconds, of each lesson segment is represented at the bottom of the figure.

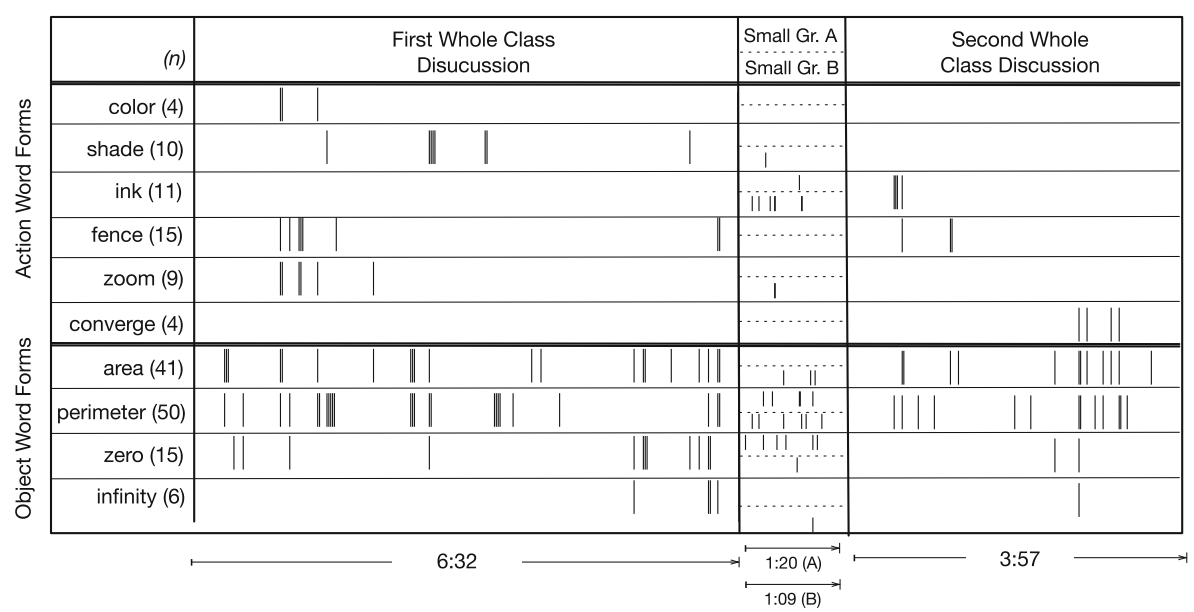

Fig. 7 Timelines displaying occurrences of action and object word forms that become constitutive of the linguistic register 
The timelines in the upper panel of Fig. 7 (Action Word Forms) show that participants used action word forms with varying frequencies, from four times for converging and coloring to 15 times for fencing. The timeline also shows varied onset and offset times in the introduction and use of each action word form. Participants used coloring only in the first whole class discussion (to indicate subtraction of area through Sierpinski transformations), and then did not use it again; perhaps the early offset time in coloring was related to the onset of shading, a word form with a similar sense. In contrast, participants never used inking or converging in the first whole class discussion: Inking first appeared in the discussion of small group A and continued to be used in the last whole class discussion; converging appeared only towards the end of the last whole class discussion. Fencing was the only key word form used in both of the whole class discussions.

The timelines in the lower panel of Fig. 7 (Object Word Forms) show that participants used object word forms with greater frequency than action word forms. References to geometrical objects, area and perimeter, were frequent, occurring 41 and 50 times, respectively, providing evidence that these objects were central to the discussion and participants' conjectures for all stages in the Sierpinski triangle construction. Numerical objects zero and infinity were used much less frequently, perhaps because they are more pertinent to one strand of the inductive reasoning process (considering the end state of Sierpinski construction and not the process of construction). Both zero and infinity were used more frequently in the first whole class discussion than in other lesson segments; zero was more frequently displayed than infinity, perhaps due to zero's use to consider end states of both perimeter and area, whereas infinity was constrained to the end state for perimeter only (none of the participants thought that area would go to infinity).

As further evidence of the emerging linguistic register in the Conjectures Discussions, we also examined the number of participants who used each of the focal action and object word forms. Figure 8 displays these data and provides evidence of the reproduction of forms constituting the linguistic register by multiple classroom participants. The object word forms were reproduced by many participants in the Conjectures Discussions. Perimeter was used by every person who participated in the Conjectures Discussions, and area and zero were used by a majority of participants (eight and five respectively). Infinity was used by only three participants. None of the key forms that we analyzed was used by only a single participant. The action word forms we consider received varied uptake across the classroom community. Shading and zooming were taken up and used by four participants in the classroom of nine participants (excluding a co-instructor and two students who did not take part in the Conjectures Discussions) and were used across multiple lesson segments (small group and whole class). Other action word forms, such as coloring, inking, and converging, showed more limited reproduction across participants, each only used by two.

In summary, the action and object word forms that we documented over the course of the Conjectures Discussions show variability in frequency of occurrences, as well as variability in uptake by participants. Some forms were used early in the Conjectures Discussions with either little subsequent use or a great deal of subsequent use. Some forms emerged mid discussion, occurred frequently, but were used by only two individuals, while still others showed sparse use throughout or dense use throughout. We regard the variability to be a likely characteristic of linguistic registers. Our 


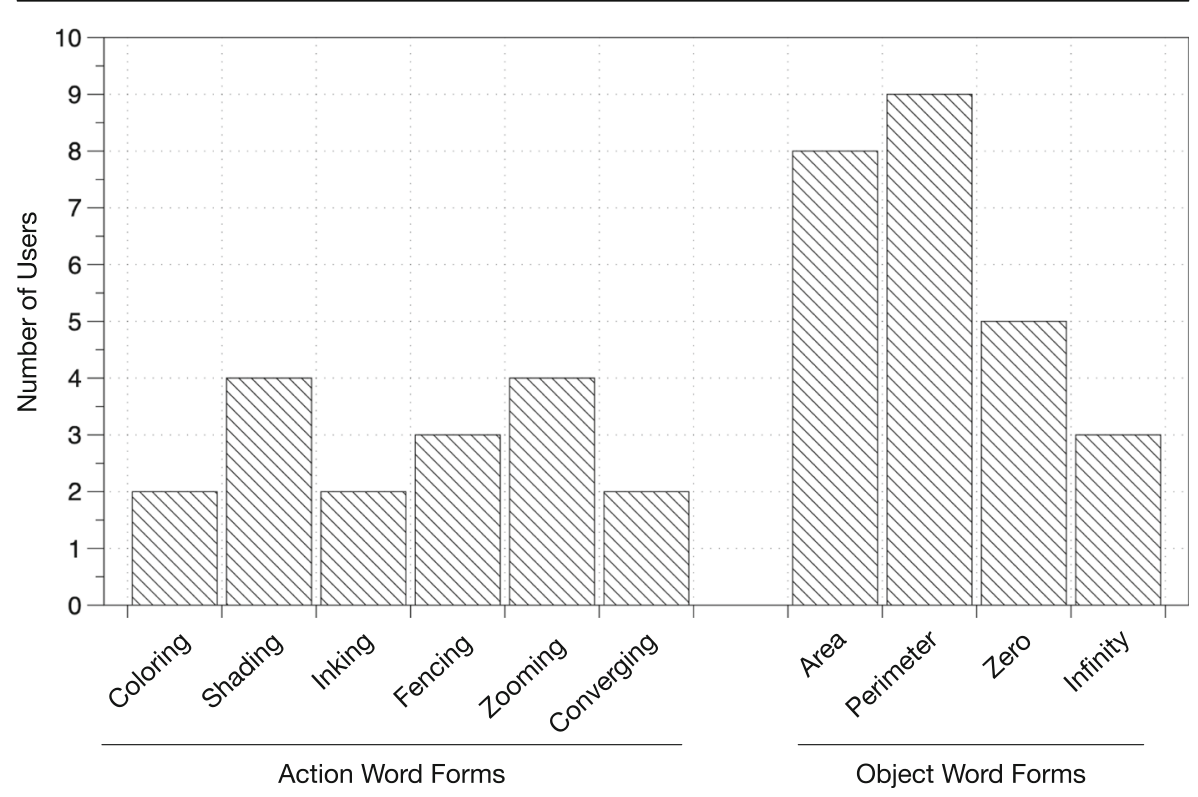

Fig. 8 The number of individuals who use each action word form at least once during the Conjecture Discussions

documentation of the emerging linguistic register provides the context for our subsequent developmental and cultural analyses.

\section{Developmental Analyses of Form-Function Relations Constituting the Register}

This section focuses on developmental analyses of the emerging linguistic register. We analyze microgenetic processes as participants schematize form-function relations, sociogenetic processes in the emerging autonomy of the linguistic register, and ontogenetic processes as individuals use and re-use form-function relations over the course of their own short-term development during the Conjectures Discussions.

\section{Microgenetic Processes}

We found that word forms constituting the linguistic register served different functions. In their communicative exchanges, participants schematized action word forms, like fencing, to conceptualize changes in area and perimeter across the Sierpinski transformations as they engaged in inductive reasoning. In our introduction, we noted some of the functions that action word forms could serve for inductive reasoningunderstanding base stages, successive stages, and end state of the Sierpinski triangle. Now consider the referential functions that object word forms could serve.

To illustrate the referential functions that object word forms served in participants construction of form-function relations, recall Carmen's excerpt cited earlier, "It's going to be black, so there's no area..." In this case area appears to refer to a geometric 
object with a material property, as though it is colored in with the felt-tipped marker. On other occasions, we find that participants will use the word form area to refer to idealized objects, as when a participant refers to the Sierpinski area as an object that undergoes transformations to a hypothetical limit (cf. de Kirby \& Saxe, 2014). These different ontological properties had different consequences as participants engaged in the microgenesis of form-function relations. For example, in Carmen's case, the material treatment of area implies that at the end state, perimeter resolves to zero.

Action Word Form-Function Relations To identify the functions served by key action word forms in the Sierpinski classroom, we analyzed the video recordings and identified three broad types of Sierpinski-linked functions, each central to inductive reasoning. As we noted earlier, participants could use action word forms to support reasoning about (i) the structural consequences for area and perimeter at a base stage of their geometrical constructions - connecting the midpoints of bisected sides of an equilateral triangle and then removing the middle triangle; (ii) changes in area and perimeter across subsequent self-similar constructions; and (iii) the end state for area and perimeter of the constructive process. ${ }^{2}$ An inductive argument might involve each of these strands as follows: (i) when a piece is removed from the initial triangle, the area of the shape is reduced, (ii) when this is done over and over again, the area of the shape continually decreases, so (iii) by the end, the final shape must have no area.

For our analyses of form-function relations, we coded all displays of key action word forms in the Conjectures Discussions with regard to whether participants structured them to serve functions related to (i) base stage, (ii) successive stages, or (iii) end state of inductive reasoning. Displays that did not clearly serve any of these inductive functions were coded as "other" (see Appendix, Table 1 for the coding scheme.) We reached agreement on the assignment of all codes; for one portion of the video recordings, we coded word forms jointly, and, for the remainder, we coded turns independently and negotiated any disagreement to reach consensus.

Participants recruited action word forms to serve induction-related functions (base stage, successive stages, or end state) in $83 \%$ of the instances for which they were used. Figure 9 displays participants' use of these word forms and the inductive functions that they were structured to serve during the Conjectures Discussions. The figure reveals that five of the six forms were used to serve two functions, none of the forms was structured to serve all three functions, and, one form, converging, was used to reason only about the end state. Reciprocally, particular functions were served by more than one form. For example, coloring, fencing and converging were all used in relation to the end state of the Sierpinski triangle, while shading, inking, and zooming were each used to reason about a base stage. In conceptualizing change across successive stages, participants used all word forms but converging.

The following sections provide a closer look into participants' microgenetic activity as they structured action word forms to serve inductive functions.

\footnotetext{
${ }^{2}$ Each of these induction-related functions can have multiple variants, and we cannot address them all in this article. We also note that the strands of inductive reasoning identified here are not meant to serve as a model for mathematical induction writ large, but as aspects of students' reasoning in the context of the Sierpinski problem.
} 


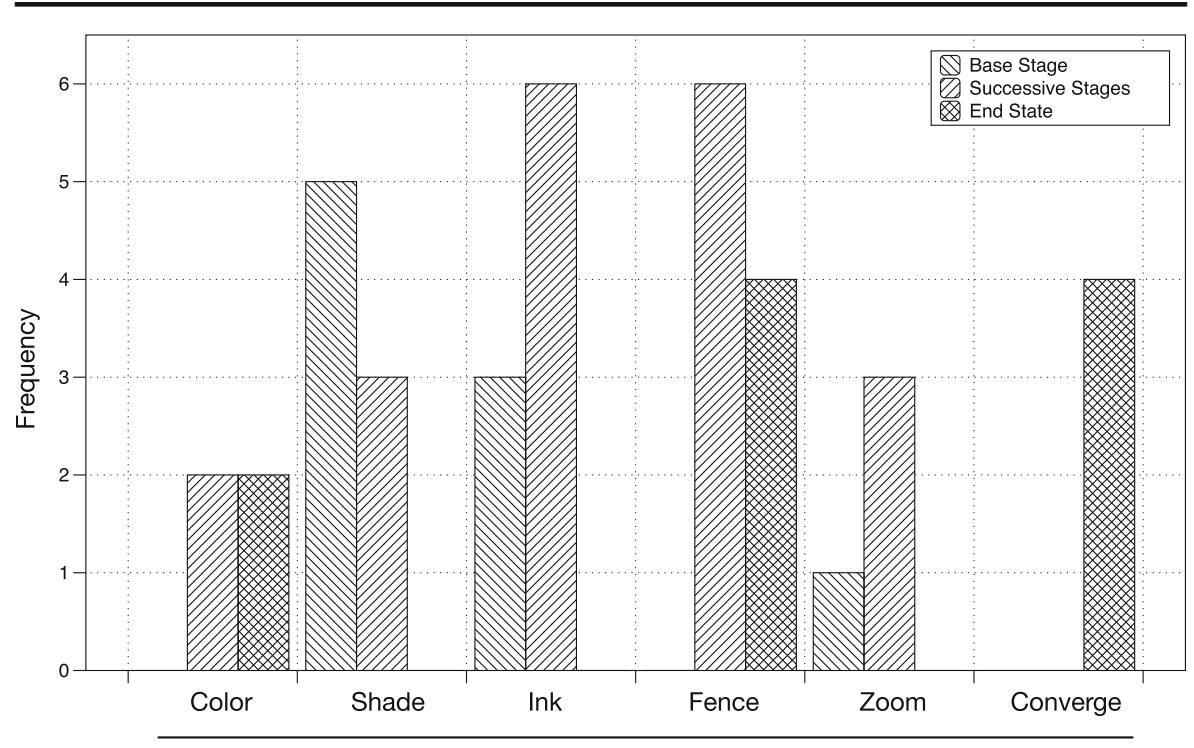

Word Forms

Fig. 9 Participants' generation of form-function relations for action word forms during the Conjectures Discussions

Base stage. At the crux of inductive reasoning is isolating and understanding the structure of a base stage: connect the midpoints of an equilateral triangle's sides and remove the middle triangle. Participants structured key word forms to serve functions related to reasoning about a base stage in the inductive process.

To identify and understand structural properties of the Sierpinski procedure requires a microgenetic act, which could take many different guises. On the one hand, a student participant might not identify a base stage at all (not differentiating a base stage from the iterative process of successive constructions) or might identify the recursive procedure (specified in Fig. 1) as important to consider, but not conceptualize inverse relations between area and perimeter or not conceptualize area and perimeter in ways intended by the instructor. On the other hand, a participant might understand the geometrical construction procedure resulting in a diminished area (due to removal of the middle) and increased perimeter (due to additional drawn lines). Yet other approaches could be arithmetic. For example, a student might reason that the resulting construction is three-fourth the area of the original, since the original is partitioned into four parts of equivalent area and one is removed; or a student might reason that the resulting construction is $3 / 2$ of the perimeter of the original, since three sides of the original were partitioned into 6 equivalent lengths, and in the process, three equivalent lengths were added, resulting in nine-sixths of the original length.

Consider how Kevin, during the first whole class discussion, structured shading ("shade it in") as he reasoned about area and perimeter in a base stage.

Kevin: So what... So the, the perimeter is... also can be considered the perimeter of the black. Part of the perimeter is the perimeter of the black. 'Cause see, when you... When you shade it in, you're adding the perimeter of the black. 
In Kevin's microgenetic construction, he used shading to both conceptualize and communicate about simultaneous reduction in area and increase in perimeter at a base stage. Shading is not merely an expression of a preformed worked out idea, but he used the shading form in concert with an insight to serve a base-stage function through, in Werner and Kaplan's terms, a schematizing process. He used when you shade it in to crystallize the intrinsic structural relation between the removal of area and the addition of perimeter. In Kevin's microgenetic act, shading served a generative function, constitutive of his reasoning about the structural properties of the Sierpinski transformation.

Successive stages and end state. Like conjectures about a base stage, participants' reasonings about change in area and perimeter across successive stages and about the end states of area and perimeter were also microgenetic acts. Consider the use of fencing in an interaction between Carmen, the Instructor, and Joy. Carmen used fencing to reason about the end state of construction of the Sierpinski triangle, while Joy used the same form to reason about change across successive stages of construction.

Carmen: I was thinking if we keep zooming... Okay, for our area thing, we were going to keep zooming in, keep coloring in, so eventually we're gonna color all in. It's going to be black, so there's no area, so there's nothing to... No area there's nothing to put a fence around it. So, there'd be no perimeter... and then

Instructor: So Carmen is thinking that the perimeter then would be zero, because there's no... There's nothing left to put a fence around. And Joy, you were thinking what?

Joy: I kind of thought it's toward the opposite end-like, if you zoom in there's more to fence, and if you zoom in there's more to fence, and you just keep putting in more fencing material, because as you zoom in there's more and more to fence. Until... Except that you'd fill in the triangle, to some extent.

In her comment, Carmen used fencing as a material metaphor to represent perimeter, structuring it to reason about the final state of the Sierpinski Triangle: Since there is no area at this stage, she reasoned, there is nothing to put a fence around and thus no perimeter. In contrast, Joy used the fencing metaphor as perimeter to serve the function of reasoning about successive stages: Since more fencing material is needed from one stage to the next, the perimeter must increase accordingly. Carmen and Joy's differing paths for structuring fencing in their microgenetic constructions led to opposing conjectures. While Carmen concluded that "there'd be no perimeter", Joy concluded that perimeter would increase until "you'd fill in the triangle, to some extent."

Object Word Form-Function Relations Our analysis of how participants schematized object word forms in the context of the Conjectures Discussions focused on area and perimeter. These word forms supported reference to two kinds of objects - material objects or idealized objects. As we noted earlier, material objects have physical properties - a drawn line has width whereas an idealized line is one dimensional. Thus using perimeter to refer to a material object (as if the perimeter line were a boundary with its linear segment containing both length and breadth) leads to a baffling lack of 
differentiation between area and perimeter over the course of Sierpinski transformations, as in the case of Elise's comment.

Elise: ...you color it all in so it's all black, but... What you're coloring in, is perimeter, to some extent. Not totally, because it's also area...

To analyze students' referential functions for area and perimeter-material vs. ideal interpretation - we coded all instances of their use during the Conjectures Discussions (see Appendix, Table 2 for the coding scheme.) For those instances in which the use of area or perimeter was judged to be ambiguous, we coded them as such. For one portion of the sample, we coded turns jointly. For the remainder, we coded turns independently and reviewed any disagreements, achieving $100 \%$ agreement after discussion. Figure 10 contains each reference to area and perimeter (both frequencies and moments of occurrence) as well as whether its affordance supported a material interpretation, an ideal interpretation, or whether the affordance was judged to be ambiguous.

Figure 10 reveals that participants recruited area and perimeter to refer to material or ideal objects in $69 \%$ of the instances in which they were used, while $31 \%$ of their uses of these word forms were ambiguous in function. The "ambiguous" uses may indicate occasions when participants do not differentiate between material and ideal representations when they use a material representation as an icon for an idealized conceptual referent (Peirce, 1955). Or, of course, the functions may be ambiguous due to limitations in our access to participants' thinking.

Figure 10 also reveals that material and ideal referential functions were frequent in the Conjectures Discussions. Material interpretations were common-44\% of references for perimeter and $34 \%$ of references for area. The frequency of material

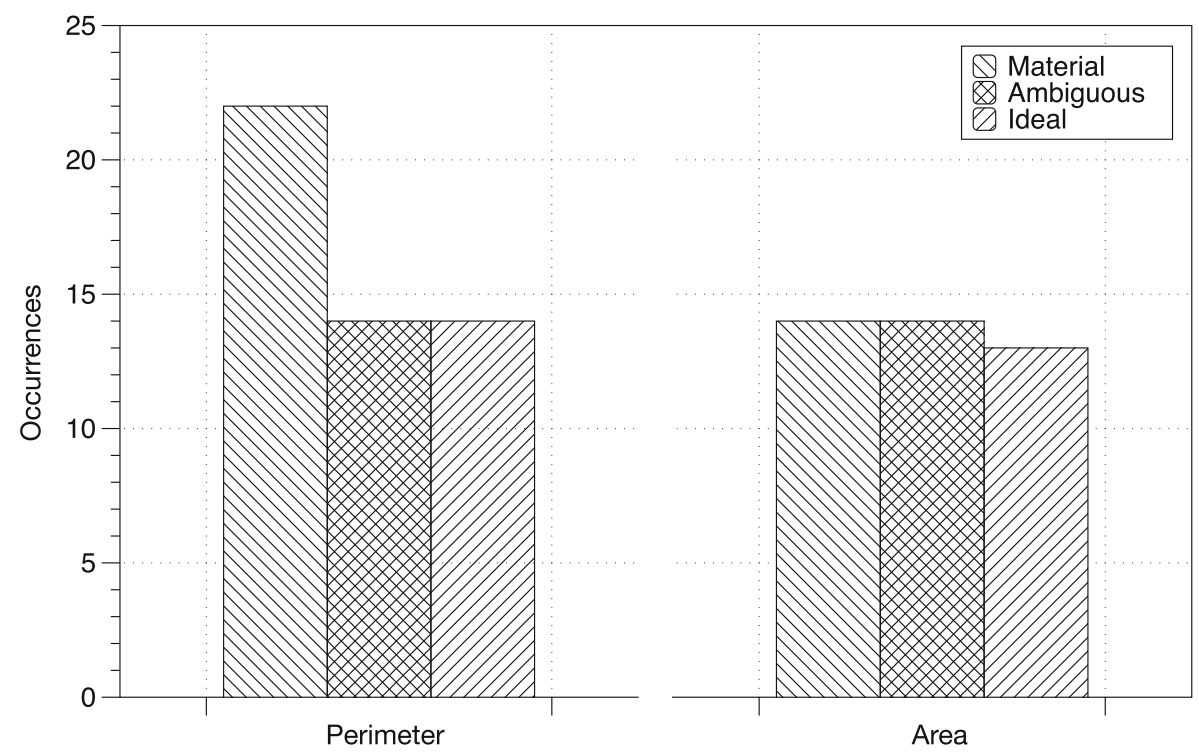

Fig. 10 Participants' generation of form-function relations for object word forms during the Conjectures Discussions 
interpretations likely reflects the 20 minutes students spent with their material constructions in the "Preparatory for Conjectures Discussions activity" as they followed the Sierpinski procedure (see Fig. 3). Ideal interpretations were less common, with $28 \%$ of references for perimeter and 32\% of references for area coded as ideal. The differentiation between material and ideal was never made explicit in exchanges, but the distinction had implications for participants' conceptualizing activity in addressing the Sierpinski problem, as we noted earlier.

We now turn to analyses that reveal differences in form-function relations for area and perimeter as these word forms were used in the whole class and small group discussions. Figure 11 provides timeline representations of the use of area and perimeter and the referential functions for these forms (material or ideal) across the first whole class discussion, small group discussions of groups A and B, and the second whole class discussion.

Figure 11 reveals that, in the small group discussions, the majority of occurrences referred to a material interpretation of area and perimeter. In small group A, of the nine occurrences of area and perimeter, eight were coded as material and none were coded as ideal; in small group B, of the 11 occurrences, eight were coded as material and only two were coded as ideal. The frequent material functions for these forms, especially in small groups, may be linked to participants' close engagement with the process of construction of the material triangle itself. Indeed, the material representations of the triangles that each small group had constructed were displayed (as evident in the photograph in Fig. 3), and conjectures in the small groups were focused on and referred frequently to those material representations.

The whole class discussions contrasted with small group discussions with regard to the referential functions for area and perimeter. In the whole class discussions, $30 \%$ of the references were material, $34 \%$ ideal, and $36 \%$ ambiguous. A large portion of the ideal references made during the whole class discussions were those of the instructor; of the 16 times the instructor used area or perimeter during the two whole class discussions, only one referenced a material interpretation of the word forms. In contrast, during whole class discussions, 20 of the students' 54 (37\%) uses of these word forms referenced material interpretations, 18 were judged to be ambiguous (33\%), and 16 referenced ideal interpretations $(30 \%)$.

\begin{tabular}{|c|c|c|c|c|c|c|c|}
\hline$n$ & \multicolumn{4}{|c|}{ First Whole Class } & \multirow{2}{*}{$\begin{array}{c}\text { Small GrA } \\
\text { Small Gr B } \\
\triangle \triangle \triangle \\
\triangle \triangle\end{array}$} & \multicolumn{2}{|c|}{ Second Whole Class } \\
\hline 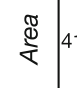 & 41 故 & $\Delta \Delta \Delta \triangle \| \triangle$ & 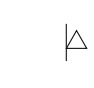 & 知证制 & & 44 & 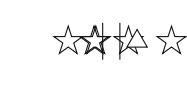 \\
\hline 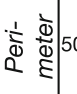 & 50 & 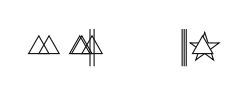 & 2 & 於 & 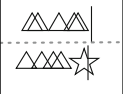 & $\Delta||^{2}$ & 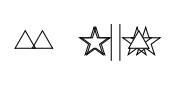 \\
\hline
\end{tabular}

Ontological affordances: $\Delta$ Material $\lesssim$ Ideal $\mid$ Ambiguous

Fig. 11 Timelines displaying ontological affordances for each occurrence of 'area' and 'perimeter' 
Summary Word forms in themselves do not contain functions. Rather they are structured by individuals to serve functions in processes of microgenesis. In our analyses of microgenetic processes, we produced evidence of the functions that participants structured forms to serve, whether action word forms to make sense of recursive Sierpinski transformations or object word forms to refer to geometrical objects with differing ontological properties. In the next section, we consider the uptake of the emerging linguistic register, that is, the extent to which it was reproduced by participants through sociogenetic processes in the Conjectures Discussions.

\section{Sociogenetic Processes}

The emerging linguistic register is a sociogenetic development, becoming constituted over the course of the Conjectures Discussions as it emerges and is reproduced and altered in the activities of multiple participants. In the prior section, Fig. 8 displayed the number of participants who produced and reproduced each of our target action and object word forms; there was variability in uptake across word forms, from perimeter, which was used by every participant in the Conjectures Discussions, to coloring, inking, and converging, which were used by only two participants each.

To illustrate sociogenetic processes in which form-function relations were reproduced and altered, we consider the use of an action word form, converging, and two object word forms, area and perimeter, in an exchange between Sam and Joy. Converging emerged late in the second whole class discussion, and all four instances of the word form are contained in this exchange.

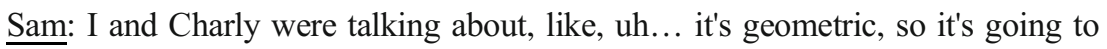
converge. So the area would be... go to zero... There would be limited amount of areas, so we're going to have a limited number of... perimeters. So we don't have infinite number of perimeters.

Joy: So my question is - does it converge to the total area of the triangle, because you can't fit anymore?

Instructor: The "it" is the perimeter?

Joy: Into the area of the perimeter?

Sam: No, the area will converge.

Joy: A perimeter... The perimeter converges, but it fills a space that is the area of the original triangle. Can you fit more perimeter into the triangle that you originally started with?

In Sam's schematizing activity, Sam structured converging to conceptualize the fate of area at the end state of the Sierpinski triangle - "it's geometric, so it's going to converge." He argued that the area of the Sierpinski triangle, represented by a "geometric" numeric sequence, would converge "to zero." Converging, for Sam, is a process applied to idealized mathematical objects, of which area is one, and Sam appeared to presuppose that Joy would interpret converging to serve the same function.

Yet Joy immediately took up converging to refer to the material representation of the triangle - "does it converge to the total area of the triangle, because you can't fit anymore?" As Joy reproduced the form introduced by Sam in his statement, she used it 
to serve a similar function, to consider the fate of the Sierpinski triangle at a hypothetical end state, but she also altered it when using it to consider the transformation of material rather than ideal forms. Both her uses of converging - "converge to the total area of the triangle" and "it fills a space that is the area of the original triangle" referenced material objects, a marked departure from the original form-function relations introduced by Sam. Thus form-function relations related to converging were introduced by Sam and subsequently reproduced and altered by Joy. This exchange marks the emergence of converging as an element of the linguistic register, a late emergent sociogenetic development.

The use of area and perimeter in this exchange also illustrates the ways in which interlocutors can talk across each other as they take up and reproduce an emergent linguistic register. Sam and Joy created different referential functions for geometrical objects-Sam, an idealized reference ("it's geometric...so the area would go to zero"), and Joy, a material reference ("you can't fit any more" and "it fills a space"). Further, while Sam was referring to area ("No, the area will converge"), Joy appeared to be referring to perimeter ("the perimeter converges"), though this crucial difference was obscured by her use of the third person singular, "it."

The interaction was in some ways coherent in that Joy and Sam appeared to be responsive to one another's communications related to end state. But the common ground is partial and only taken-as-shared, because Sam and Joy were talking about different categories of geometrical objects, idealized and material. Sam was talking about area, and Joy was talking about perimeter; Sam appeared to focus on area converging to its limit zero, and Joy appeared to focus on perimeter converging to area. Sam's and Joy's exchange highlights the difficulty for interlocutors, both of whom may be making use of a common linguistic register, to read intended meanings through one another's communicative displays in a sociogenetic process.

In summary, the reproduction of action and object word forms that serve Sierpinskilinked functions by multiple classroom participants is a sociogenetic process that supports the emergence of a linguistic register. In participants' reproduction and alteration of forms that serve specialized functions, form-function relations become detached from any single individual's use and become part of the collective representations used in the classroom community. Still missing in our analysis is how these forms become constitutive of the ontogenetic developments of individuals through their participations in the Conjectures Discussions. We address this below.

\section{Ontogenetic Processes}

Our analyses of ontogenetic developments focus on individual participants' shifts in form-function relations over the course of the Conjectures Discussions. As we noted earlier, the short duration of the Conjectures Discussions limited our opportunity to observe ontogenetic shifts. That stated, our analyses illustrate an analytic direction that we regard as useful in understanding individual development through participation in collective practices. In our analyses, we consider whether participants extend the use of a form previously elaborated to subsequently serve new functions, thus displaying continuity of form and discontinuity of function over the course of the Conjectures Discussions. Conversely, we consider whether participants adopt new forms to serve earlier emerging functions, a 
discontinuity in form but continuity of function over time. In our analyses, we tracked shifts in form-function relations of participants in the Conjectures Discussions. We present the illustrative cases of four participants here- the instructor, Carmen, Joy, and Sam - each of whom showed different ontogenetic trajectories. The forms we tracked included fencing, inking, zooming, and converging, and were selected because ontogenetic analysis of these forms revealed interesting variation.

Figure 12 contains timelines representing induction-related functions served by the four word forms that we tracked in four participants. The number of times each individual uses each word form is indicated on the left of the timeline (n). The functions are supports for conceptualizing the three stages in inductive reasoning: (a) base stage - an empty triangle; (b) successive stages - a white triangle containing an inverted black triangle; (c) end state - a triangle containing multiple inscribed triangles.

The timelines reveal that, over the course of the Conjectures Discussions, some participants used a given word form consistently to serve the same

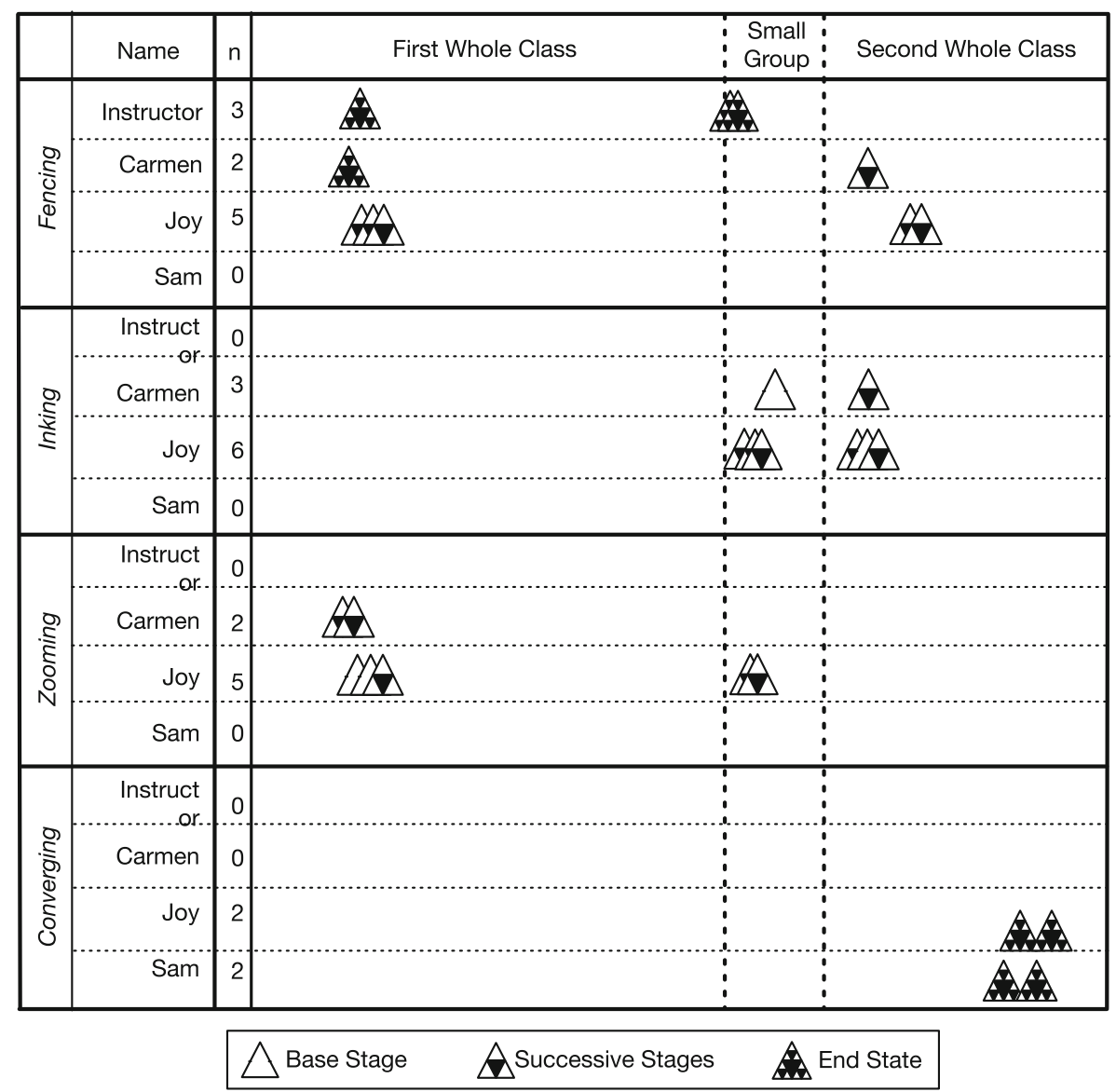

Fig. 12 Timelines displaying induction-related functions served by key action word forms by individuals 
function, while others extended word forms to serve new induction-related functions. Joy used fencing to serve a successive stages function in both the first and last whole class discussion, evidence suggesting no ontogenetic shifts, at least in this limited data sample. In contrast, other participants showed shifts of forms to accomplish new functions, for example, from successive stages to an end state. At the beginning of the first whole class discussion, Carmen used fencing to reason about an end state in the construction of the Sierpinski Triangle, while later in the second whole class discussion, she used the same word form to reason about changes across successive stages. As another example, in the first whole class discussion, Joy used zooming to refer to a base stage a couple of times, and then, in small group discussions she extended the use of zooming to reason about successive stages. When individuals used a familiar form to serve a novel function, they used what they understand to conceptualize and re-conceptualize the Sierpinski problem.

Figure 12 also provides examples of continuity of function but discontinuity of form. For example, Joy used zero (not shown in Fig. 12) to reason about an end state in the first whole class discussion, but, in the second whole class discussion, she recruited converge, a form not previously used, to serve a similar end state function, structuring a new form-function relation.

In summary, our analysis of ontogenetic processes of form-function relations linked to the register complements the microgenetic and sociogenetic processes documented earlier. Participants' protracted engagement with a problem or recurring engagement with similar problems led them to using prior forms in new ways or recruiting new forms to support functions that they had previously structured. Our ontogenetic analyses documented continuities and discontinuities in participants' sense making efforts over the course of the Conjectures Discussions.

\section{Cultural Analyses of Collective Practices that Support the Emergence, Reproduction and Alteration of the Linguistic Register}

We now turn to our complementary cultural approach to analyze the Conjectures Discussions with a focus on processes that support the emergence, reproduction, and alteration of the linguistic register. We focus on collective practices as microcosms of cultural phenomena.

We consider the whole class and the small group discussions as two kinds of collective practices during the Conjectures Discussions, each with a different normative participation structure, i.e., an emergent social organization supported by participants' expectations about who can participate and how. Both participation structures reflected an inquiry-oriented teaching/learning approach that supports values such as the importance of engaging student curiosities, building on student thinking, and fostering student agency in their learning.

\section{Who Participated?}

Figure 13 provides evidence of differing participation structures in whole class and small group discussions with regard to who participated and the extent of their participation. The figure consists of three panels, the left for the instructor, the middle 
for students in Small Group A (Carmen, Joy, and Jen), and the right for students in Small Group B (Elise, Kevin, and Mia). Each panel displays the proportion of turns for participants in the first whole class discussion, the small group discussions, and the second whole class discussion. We used separate number-of-turn denominators for each lesson segment proportion (first whole class discussion, $n=56$; small group discussion A, $n=36$; small group discussion $\mathrm{B}, n=18$; and second whole group discussion, $n=38$.) Three observations are particularly noteworthy with regard to participation.

1. The instructor was a major participant in whole class but did not participate in small group discussions. The left most panel of Fig. 13 shows that the instructor contributed significantly to both the first and the second whole class discussions: $35 \%$ of the total number of turns in the first whole class discussion and $23 \%$ of the total number of turns in the second. These are substantial percentages since the instructor constitutes one of ten participants in these discussions.

2. Every student participated in small group discussions, but not all participated in whole class discussions. The middle and right panels of Fig. 13 shows the proportions of turns that students took in the first whole class discussion, their small group discussions (A or B), and the second whole class discussions. Every student in the two small groups participated in the small group discussions, but two students (Kevin and Mia) did not participate at all in the second whole class discussion.

3. Every student participated to a greater extent in small group than in whole class $\underline{\text { discussions. The middle and right panels of Fig. } 13 \text { also reveal that every student }}$ participated more frequently in the small group discussions than in whole class discussion. Further, for all but one student (Joy), there was a several fold increase

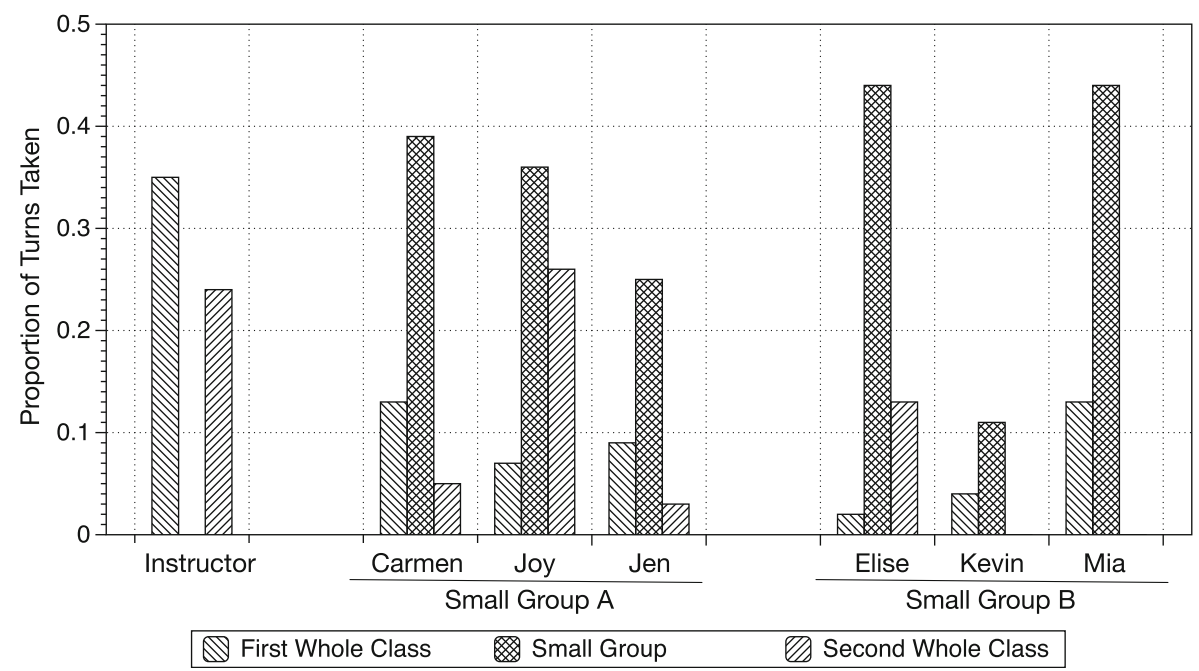

Fig. 13 Relative proportions of instructor's and small group participants' turns during whole class and small group discussions 
in their proportion of turns in small group discussions compared with either whole class discussion.

The differing organizations for participation are marked in whole class and small group discussions, with differences in leadership and opportunities to participate. These differences mark different opportunities to schematize form-function relations in public displays as well as different kinds of opportunities to make sense of others' schematizing activities.

\section{How Did Individuals Participate?}

Regarding the how? of participation, we examine the social positions participants took up, the norms for listening to one another's public displays, and the norms for explaining one's thinking in public displays. The focus is on how participants' use of the emerging linguistic register becomes at once constitutive of collective practices with a focus on patterns of social positioning and norms, and at the same time, how the collective practices support participants' construction of the linguistic register.

Positioning The instructor's position as orchestrator of whole class discussions is evident in the high percentage of turns that contained a request of and/or a question for students about their thinking; $90 \%$ of his turns, on average, contained a question or request (see Appendix, Table 4 for the coding scheme.) By "on average" we mean the total number of the instructor's questions/requests divided by the total number of the his turns. Some of the instructor's requests were for students to explain their own thinking, such as

Instructor: So... Carmen, Joy and Jen, tell us... Tell us what your table... Maybe you don't have a final conjecture but tell us what you're thinking now...

Other requests were for students to comment on or revoice the thinking of their peers, such as

Instructor: Curtis, you were nodding your head when Kevin was talking. Can you say a little about what you interpreted Kevin to say?

Instructor: Soo, can you explain with your own words, what this conversation is about, between Kevin and Carmen?

Still other requests were for students to clarify ideas, such as

Instructor: Are you sure?

Instructor: You think so?

While the instructor positioned students by making requests for public displays, reciprocally, students displayed their thinking, often making use of forms to serve functions constituting the emerging linguistic register. For example, when the instructor called on Elise, she stated and questioned, 
Elise: So, what I feel, like, what Carmen's saying is when you zoom in... Or she says you color it all in so it's all black, but... What you're coloring in, is perimeter, to some extent. Not totally, because it's also area. But, like, every time you build a little triangle, you have more perimeter in there, right? So, then, all those... I don't know... Does all the black become all the tiny little pieces of all the tiny triangles?

In her response, Elise took up the position of a student in an inquiry-oriented classroom, thinking aloud as she considered her ideas, building on others' contributions, and posing inquiry-oriented questions.

Norms for listening The instructor played an active role in supporting norms for listening. In about a quarter of his questions and requests, the instructor asked students to explain or respond to each other's ideas with questions like "So, can you explain with your own words, what this conversation is about, between Kevin and Carmen?" and "Mia, you're agreeing more with Joy?" The instructor also requested students to revoice one another's ideas with indirect requests like, "Curtis... Can you say a little about what you interpreted Kevin to say?" By his frequent requests for students to take responsibility for listening to their peers, the instructor supported a community in which students' contributions were understood to be important (see Appendix, Table 3 for coding scheme.)

Students' actions were generally consistent with the expectation that they would focus on one another's contributions and take on the role of active listeners. They revoiced, commented on, or extended the ideas of their peers in nearly half of their turns (44\%), both in whole class discussions when the instructor was present (where $35 \%$ of turns referenced prior contributions), and in the small groups (where $41 \%$ of turns referenced prior contributions). Evidence that the norm for active listening was taken up in small group work includes common references to others' ideas; in Small Group B, participants referenced one another's ideas on $61 \%$ of their turns, and in Small Group A, participants referenced one another's ideas on $31 \%$ of turns.

Though the focus on listening to ideas of others was supported in both the instructor's and students' contributions, there was also evidence of differential attentiveness by participants to some students' contributions over others. To analyze differential attentiveness to one another's displays, we produced an index of participants' focus of attention in their public displays by counting the number of times a participant was explicitly referred to in the third person in talk, such as "I understand Carmen." Note that we excluded turns in which a participant was referred to by name in the second person, such as a named, direct request, "Carmen, tell us about your idea." These were less relevant for our analysis as they did not appear to confer the same mark of status.

Figure 14 displays each participants' total number of turns (left panel) as well as the number of turns in which they were referenced (right panel) in the Conjectures Discussions. The number of references to participants (right panel) ranged from zero to 6 . Attentiveness was not merely due to the frequency of particular students' contributions; the extent of students' contributions and the number of times they were referenced were uncorrelated (Spearman rank order correlation, $\mathrm{r}(N=9)=.017, p>.05$ ).

Norms for public displays of thinking We found considerable evidence in the lesson of a norm that students should contribute a public display of their thinking. The 
instructors' questions and requests provided ample support for the claim. Consider some of the statements by the instructors in pre- Conjectures Discussions activity as well as during the Conjectures Discussions.

Just prior to the Conjectures Discussions, the co-instructors made comments that supported students' engagement with reasoning about the Sierpinski problem as well as seeding the expectation that they should be prepared to share their thinking.

Instructor: ...take a minute in your groups and come to some sort of... sort of like tentative conclusion. Don't do any computations, just come to some tentative conclusions about area and perimeter, and then share with the rest of the class.

Instructor: Without computation. Just sort of conceptually what makes sense for you.

During the Conjectures Discussions, the instructor asked open-ended questions about student thinking. His questions and requests were frequently open ended, eliciting students' mathematical reasoning about the Sierpinski problem and about their peers' ideas; 17 out of his 27 turns contained a request for students to explain their own thinking or that of their peers (see Appendix, Table 3 for the coding scheme.) Also, during the Conjectures Discussions, the instructor did not ask any closed questions, i.e., questions that would elicit a single numerical response that could be evaluated as correct or incorrect.

The norm for public displays of student thinking has implications for our treatment of the linguistic register. Students' public displays often included students' schematizing object and action word forms to serve Sierpinski-linked functions. These public displays thus were occasions for the emergence, reproduction and alteration, of the linguistic register.

In summary, in the collective practices constituting the Conjectures Discussions, we found an arena that supported substantive exchanges about mathematical

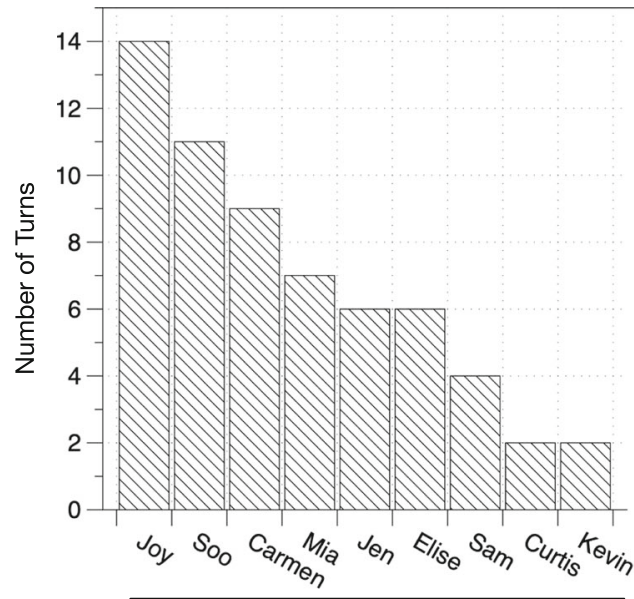

Turns by participant in whole class discussions

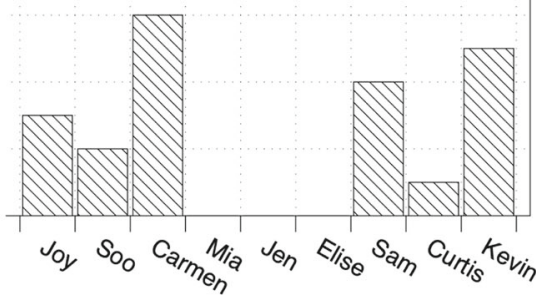

Turns referencing participant in whole class discussions

Fig. 14 Turns taken by each participant as contrasted with turns referencing each participant in whole class discussions 
ideas often involving the linguistic register. Key features of the collective practices were the social positions that participants took up, the norms for listening, and the norms for public displays of thinking. In the next section, we consider participants' efforts to generate a common ground and the role of these efforts in the emergence of the linguistic register.

\section{Generating a Common Ground-A Context for the Emergence, Reproduction, and Alteration of a Linguistic Register}

Having analyzed who participated and how, we now consider a complex phenomenon that cuts across the practices: the dynamics whereby a taken-as-shared common ground becomes constituted and reconstituted as participants generate communicative displays involving action and object word forms. We argue that efforts to establish a common ground lead to the emergence of a linguistic register related to the Sierpinski problem; reciprocally, the emergent linguistic register can be a resource for re-establishing a common ground.

The Sierpinski problem presents some unusual challenges for speakers and listeners in generating and interpreting one another's communicative displays. For example, we previously cited an exchange between Sam and Jen in our analysis of the emergence of converging as a sociogenetic development. Sam and Jen faced challenges in understanding one another's ideas as one talked about area and the other talked about perimeter. For the Sierpinski problem, students grappled with the unfamiliar ideas of geometric objects with zero area and infinite perimeter, taking mathematical limits, and reasoning by mathematical induction. It is no surprise that students appeared to lack access to linguistic conventions that could readily support their communication about the Sierpinski problem. It is in participants' efforts to address the challenges of generating a common ground that we locate a source for the emergence, reproduction and alteration of the linguistic register.

In our review of the Conjectures Discussions, we identified actions that could lead to the emergence, reproduction and alteration of the register as participants worked to establish a common ground. Participants (i) revoiced and built on each other's contributions, and (ii) asked questions and produced repairs by formulating explicit agreements about the functions of word forms in the emerging register.

Revoicing and building on the contributions of others As he orchestrated classroom discussions, the instructor often "revoiced" students' contributions. Such acts of revoicing included re-phrasing, summarizing, and sometimes modifying students' statements (Flood, 2018; Forman et al., 1998; Kwon et al., 2008; O'Connor \& Michaels, 1993). Often the instructor's acts of revoicing reproduced word forms constituting the linguistic register, and given the instructor's privileged status in the classroom, his use of the register may have supported students' further reproduction of the register (see Saxe, 2012 for a related idea of "broadcast" in a treatment of sociogenesis). To determine the frequency of the instructor's revoicings, we counted each turn that included a revoicing and found that he revoiced student ideas in 37\% of his turns (see Appendix, Table 3 for the coding scheme.)

Consider the instructor's revoicing of Sam and Joy, which he used to initiate small group discussions and point the class towards a common ground. 
Instructor: Okay, so what Sam is saying - like, you see the perimeter is going off into infinity, like Joy is saying, but then you haven't any area, so then... That was Joy's point originally, that you, that you don't have any fence, you don't have anything to fence, so... take a minute in your groups, and come to some sort of... sort of like tentative conclusion.

The instructor took up word forms included in the register, such as area, perimeter, infinity and fence to serve Sierpinski-linked functions. Through his revoicing, he lent legitimacy to those form-function relations, supporting their further propagation in subsequent discourse in a sociogenetic process.

It was not only the instructor who revoiced students' contributions. As we previously noted, the instructor asked students to revoice one another's ideas, providing opportunities for clarification or repair of knowledge of one another's thinking. One sixth of the instructor's turns contained, on average, a request for students to revoice the thinking of others. Consider Soo's revoicing of Joy's contribution, at the request of the instructor,

Soo: Umm... I think... Umm... Joy is saying, like, you keep zooming in you're going to get more triangles forming, so you have more areas, so you keep adding the numbers, right?

Or Curtis's interpretation of Kevin's contribution, in response to the instructor's request,

Curtis: Kevin was saying that the perimeter of the... the white is also the same as the perimeter of the... perimeter of the black part. So, since there's area... There is some area of the black...

In addition to students revoicing one another, in $33 \%$ of their turns, students commented on, extended, or reacted to other's ideas (see Appendix, Table 3 for coding scheme.) These extensions of one another's contributions often led to the reproduction and alteration of the emerging register. Consider Carmen's comment and extension of Kevin's idea,

Carmen: Oh, I see what you're saying — so it's actually, like, it's a... the fence is guarding both properties,

or Joy's contrast to Carmen's idea,

Joy: I kind of thought it's toward the opposite end-like, if you zoom in there's more to fence, and if you zoom in there's more to fence, and you just keep putting in more fencing material, because as you zoom in there's more and more to fence.

In turns such as Carmen's and Joy's, students take up form-function relations introduced by their peers, and, in so doing, they generate and regenerate a common ground of talk and action, in the process reproducing and altering an emerging register. 
Clarifications and explicit agreements Participants made efforts to anticipate, attend to, and repair breakdowns in a common ground. For some word forms in the register, there was confusion about the functions they served. Sometimes, confusion led to multiple efforts to reach explicit agreement about the referential function for the word. We illustrate a case of this with the use of perimeter.

'Perimeter' stipulated as 'circumference'? Prior to the Conjectures Discussions, a co-instructor (perhaps inadvertently) substituted the term circumference for perimeter, leading to confusion for both groups A and B. Each small group independently turned to the instructor for clarification about the meaning of circumference. He replied separately to both groups that he should have used the term perimeter. Henceforth, perimeter was substituted for circumference throughout the subsequent Conjectures Discussions.

'Perimeter' stipulated as 'cumulative perimeter'? In her turns prior to and during the Conjectures Discussions, Carmen explicitly asked for a further elaboration of the referential function for perimeter. She distinguished between what she referred to as 'cumulative perimeter' and 'perimeter', and argued that how the term was stipulated is consequential for conceptualizing and addressing the Sierpinski problem. Carmen attempted to clarify the distinction in the whole class discussion, with statements like,

Carmen: perimeter is zero - is it cumulative or a point in time? If cumulative, it continues to increase. But if it is the extent of perimeter as area reaches the limit, then perimeter is zero.

Her distinction was never taken up in either whole class or small group discussions.

'Perimeter' stipulated as 'inking' or 'fencing'? Additional clarity for the meaning of perimeter was provided with the use of action word forms. For example, the instructor used fencing as a means to clarify the meaning of perimeter in the Sierpinski case when he stated,

Instructor: So Carmen is thinking that the perimeter then would be zero, because there's no... There's nothing left to put a fence around.

Similarly, Joy used ink to stipulate a definition for perimeter when she stated,

$$
\text { Joy: Okay, so if you try... The ink is your perimeter, right? }
$$

In both cases, participants appear to support a common ground by turning the geometrical object, perimeter, into an action word form suited to support the conceptualization of the Sierpinski problem, one in which perimeter underwent transformations with each Sierpinski recursion.

In all of the instances cited, participants made efforts to clarify the meaning of perimeter as they worked to establish a common ground. In the first case, the effort was to reach an agreement about the function of the term circumference as distinct from perimeter in discourse and involved a stipulation involving a word substitution, perimeter for circumference. In the second, the import for a common ground was 
identified by a student in her conceptualization of the Sierpinski problemdifferentiating perimeter resulting from a single Sierpinski transformation and an accumulation of perimeters over repeated Sierpinski transformations. In the third case, participants used a trope - the action word forms of inking and fencing - to convey perimeter accumulating through Sierpinski transformations. Across these instances, as participants conceptualized the Sierpinski problem and worked to establish a common ground, we find the referential function of perimeter and related word forms established and re-established.

In sum, our analyses show that participants unwittingly reproduced and altered the linguistic register as they made efforts to communicate their thinking and establish and re-establish a common ground. These efforts were enabled and constrained by the norms, social positions, and participation structures constituting the collective practices of classroom life.

\section{Concluding Remarks}

In this article, we applied a cultural-developmental framework to a short video segment from an inquiry-oriented classroom engaged with a baffling problem. We showed that a linguistic register consisting of specialized word forms to serve Sierpinski-linked functions emerged through the interplay of individual and collective activity over time. To understand the interplay that led to the register's emergence and its place in the thinking of participants, we conducted complementary but integrated developmental and cultural analyses.

Our developmental analyses consisted of three parts. In our treatment of microgenesis, we showed that participants structured action and object word forms to serve functions related to inductive reasoning about area and perimeter; we further showed that participants sometimes schematized area and perimeter as idealized objects and other times as material objects, schematizations that had implications for participants' understanding of hypothetical end states of area and perimeter. In our treatment of sociogenesis, we showed how multiple classroom participants unwittingly reproduced and altered the register's form-function relations in their microgenetic activity; the sociogenetic process occurred as participants made efforts to communicate and interpret one another's communications. In our treatment of ontogenesis, we documented continuities and discontinuities in form-function relations structured by individual participants over the course of the Conjectures Discussions.

Our cultural analyses showed how developmental processes were enabled and constrained by individuals' participations in two collective practices, and at the same time, how developmental processes became constitutive of the collective practices with which individuals were engaged. We showed that participants varied in the frequency and character of their microgenetic schematizations as they took up and reproduced norms for listening and for making public displays of thinking, norms that supported both participants' microgenetic constructions as well as sociogenetic processes by which the register was reproduced and altered. Further, as they communicated, we found frequent efforts to establish and re-establish a common ground of talk and action. These efforts to revoice each others' contributions, build on each others' ideas, ask clarifying questions and come to explicit agreements, led to the emergence, 
reproduction, and alteration of the linguistic register. The emerging register, in turn, often served as a resource for participants' efforts to re-establish a common ground.

The present analyses extend prior uses of our cultural-developmental framework by integrating cultural and developmental microanalyses of face-to-face interactions within a university classroom setting, drawing on novel methodological techniques. At the same time, we note that there are several limitations to our treatment. First, while participants in the Sierpinski classroom use linguistic, figural, embodied, and computational representations in their activity, our analysis focused on select word forms and the functions they served in the Sierpinski classroom. A more complete analysis would have included a consideration of participants' gestures, written computations, and drawings, sometimes used in concert with word forms. Second, our analyses focused on a short segment of video recordings from one lesson. A more complete investigation of the reproduction and alteration of classroom norms, social positions, and participants' status could be obtained from analyses of recordings collected over the course of a longer period of time and with additional techniques for gathering evidence of cognitive developmental processes, such as interviews and sociograms. In short, our analyses lacked the extensive data sources that we have typically employed in our analyses of collective practices in and out of classroom worlds (cf. Saxe, 1991, 2012, 2015; Saxe et al., 1987; Saxe et al., 2015a, b).

While our cultural-developmental framework was used in this article to illuminate intrinsic relations between developmental and cultural processes in one classroom lesson, we argue that the approach could be usefully extended to investigate the interplay between individual and collective activity in mathematical reasoning in a variety of classroom settings, including those with didactic teaching practices. In the collective practices that emerge in all classrooms, however diverse, participation structures emerge with classroom norms and social positions; in all classrooms, participants work to generate a common ground of talk and action; and, in all classrooms, that common ground is regulated through the micro-, onto-, and sociogenesis of form-function relations. In more didactic instructional settings, however, we are quick to note that participants' thinking would not be revealed in public discourse. Additional techniques of data gathering would be needed, such as pre-lesson and post-lesson interviews, or stimulated video recall of participants' interpretations of the discourse. The framework and methodology presented in this paper could thus be extended to investigations of developmental processes and collective practices of classroom life, as well as their intrinsic relations.

Supplementary Information The online version contains supplementary material available at https://doi.org/ 10.1007/s40753-021-00151-y.

Acknowledgements This special issue has been conceived at a workshop supported by the Israel Science Foundation under grant number 2821/19. The data described above and used in the papers in this special issue have been collected in the framework of research funded by the Israel Science Foundation under grant number $843 / 15$. We are grateful to T. Dreyfus, M. Tabach, and C. Rasmussen for providing the video episode that we analyzed in this article. For further discussions of the research that led to the video record, see Dreyfus et al., 2018; Hershkowitz et al., 2014; Tabach et al., 2014.

Code Availability Not applicable. 
Funding During the preparation of the manuscript, Amelia M. Farid was supported by a National Academy of Education/Spencer Foundation Dissertation Fellowship.

Data Availability Not applicable.

\section{Declarations}

\section{Conflict of Interest None.}

Open Access This article is licensed under a Creative Commons Attribution 4.0 International License, which permits use, sharing, adaptation, distribution and reproduction in any medium or format, as long as you give appropriate credit to the original author(s) and the source, provide a link to the Creative Commons licence, and indicate if changes were made. The images or other third party material in this article are included in the article's Creative Commons licence, unless indicated otherwise in a credit line to the material. If material is not included in the article's Creative Commons licence and your intended use is not permitted by statutory regulation or exceeds the permitted use, you will need to obtain permission directly from the copyright holder. To view a copy of this licence, visit http://creativecommons.org/licenses/by/4.0/.

\section{References}

Allan, K. (2013). What is common ground? In A. Capone, F. Lo Piparo, \& M. Carapezza (Eds.), Perspectives in Pragmatics, Philosophy, \& Psychology (Vol. 2, pp. 285-310). Springer. https://doi.org/10.1007/978-3319-01014-4_11.

Blumer, H. (1969). Symbolic Interactionism: Perspective and Method. Prentice-Hall.

Clark, H. H. (1985). Language use and language users. In G. Lindzey \& E. Aronson (Eds.), Handbook of social psychology (3rd ed., pp. 179-231). Harper and Row.

Cole, M., \& Hatano, G. (2007). Cultural-historical activity theory: Integrating phylogeny, cultural history, and ontogenesis in cultural psychology. In S. Kitayama \& D. Cohen (Eds.), Handbook of cultural psychology (pp. 109-135). The Guilford Press.

Croft, W. (2000). Explaining language change : An evolutionary approach. Longman.

de Kirby, K., \& Saxe, G. B. (2014). Using geometrical representations as cognitive technologies. Journal of Cognition and Culture, 14(5), 401-414. https://doi.org/10.1163/15685373-12342134.

diSessa, A. (2017). Knowledge in pieces: An evolving framework for understanding knowing and learning. In T. G. Amin \& O. Levrini (Eds.), Converging perspectives on conceptual change: Mapping an emerging paradigm in the learning sciences. Routledge.

Dreyfus, T., Rasmussen, C., Apkarian, N., \& Tabach, M. (2018). The complexity of knowledge construction in a classroom setting. Proceedings of the second conference of the International Network for Didactic Research in University Mathematics, Kristiansand, Norway.

Flood, V. J. (2018). Multimodal Revoicing as an interactional mechanism for connecting scientific and everyday concepts. Human Development, 61(3), 145-173. https://doi.org/10.1159/000488693.

Forman, E. A., Larreamendy-Joerns, J., Stein, M. K., \& Brown, C. A. (1998). "You're going to want to find out which and prove it": Collective argumentation in a mathematics classroom. Learning and Instruction, $8(6), 527-548$.

Grice, H. P. (1989). Studies in the way of words. Harvard University Press.

Harré, R. (2012). Positioning theory: Moral dimensions of social-cultural psychology. In J. Valsiner (Ed.), The Oxford handbook of culture and psychology. (pp. 191-206). Oxford University Press.

Hershkowitz, R., Tabach, M., Rasmussen, C., \& Dreyfus, T. (2014). Knowledge shifts in a probability classroom: A case study coordinating two methodologies. ZDM, 46(3), 363-387. https://doi.org/10. 1007/s11858-014-0576-0.

Hirvonen, P. (2016). Positioning theory and small-group interaction: Social and task positioning in the context of joint decision-making. SAGE Open, 6(3), 2158244016655584. https://doi.org/10.1177/ 2158244016655584.

Keller, R. (1994). On language change: The invisible hand in language. Routledge. 
Kwon, O., Ju, M., Rasmussen, C., Ju, M. K., Marrongelle, K., \& Park, J. H. (2008). Utilization of revoicing based on learners' thinking in an inquiry-oriented differential equations class. The Seoul National University Journal of Education Research, 17, 111-134.

Langer, J. (1970). Werner's theory of development. In P. H. Mussen (Ed.), Carmichael's manual of child psychology (Vol. 1, pp. 733-772). John Wiley \& Sons, Inc..

Lewis, D. K. (1969). Convention: A philosophical study. Harvard University Press.

O'Connor, M. C., \& Michaels, S. (1993). Aligning academic task and participation status through revoicing: Analysis of a classroom discourse strategy. Anthropology and Education, 24, 314-318. https://doi.org/10. 1525/aeq.1993.24.4.04x0063k.

Peirce, C. S. (1955). Philosophical writings of Peirce. Courier Dover Publications.

Piaget, J. (1970a). Genetic epistemology. Columbia University Press.

Piaget, J. (1970b). Piaget's theory. In P. H. Mussen (Ed.), Carmichael's manual of child psychology (pp. 703731). Wiley.

Saxe, G. B. (1991). Culture and cognitive development: Studies in mathematical understanding. Lawrence Erlbaum Associates, Inc..

Saxe, G. B. (2012). Cultural development of mathematical ideas: Papua New Guinea studies (learning in doing : Social, cognitive and computational perspectives). Cambridge University Press.

Saxe, G. B. (2015). Studying culture-cognition relations in collective practices of daily life: a research framework / El estudio de las relaciones cultura-cognición en las prácticas colectivas cotidianas: un modelo de investigación. Journal for the Study of Education and Development, Infancia y Aprendizaje, 38(3), 473-508. https://doi.org/10.1080/02103702.2015.1054669.

Saxe, G. B., de Kirby, K., Kang, B., Le, M., \& Schneider, A. (2015a). Studying cognition through time in a classroom community: The interplay between "everyday" and "scientific concepts". Human Development, 58, 5-44. https://doi.org/10.1159/000371560.

Saxe, G. B., de Kirby, K., Le, M., Sitabkhan, Y., \& Kang, B. (2015b). Understanding learning across lessons in classroom communities: A multi-leveled analytic approach. In A. Bikner-Ahsbahs, C. Knipping, \& N. Presmeg (Eds.), Doing (qualitative) research: Methodology and methods in mathematics education. Springer.

Saxe, G. B., \& Esmonde, I. (2005). Studying cognition in flux: A historical treatment of fu in the shifting structure of Oksapmin mathematics. Mind, Culture, and Activity Special Issue: Combining longitudinal, cross-historical, and cross-cultural methods to study culture and cognition, 12(3-4), 171-225.

Saxe, G. B., \& Gearhart, M. (1990). A developmental analysis of everyday topology in unschooled straw weavers. British Journal of Developmental Psychology, 8, 251-258.

Saxe, G. B., Gearhart, M., Shaughnessy, M. M., Earnest, D., Cremer, S., Sitabkhan, Y., et al. (2009). A methodological framework and empirical techniques for studying the travel of ideas in classroom communities. In B. Schwartz, T. Dreyfus, \& R. Hershkowitz (Eds.), Transformation of knowledge in classroom interaction (pp. 203-222). Routledge.

Saxe, G. B., Guberman, S. R., \& Gearhart, M. (1987). Social processes in early number development. Monographs of the Society for Research in Child Development, 52, serial no. 162. https://doi.org/10. $2307 / 1166071$.

Sfard, A. (2008). Thinking as communicating: Human development, the growth of discourses, and mathematizing. https://doi.org/10.1017/CBO9780511499944.

Slobin, D. I. (1996). From "thought and language" to "thinking for speaking." (rethinking linguistic relativity.). Cambridge University Press.

Slobin, D. I. (2003). Language and thought online: Cognitive consequences of linguistic relativity. In D. Gentner \& S. Goldin-Meadow (Eds.), Language in mind: Advances in the study of language and thought (pp. 157-192). MIT Press.

Sperber, D., \& Hirschfeld, L. (1999). Culture, cognition, and evolution: Introduction. In R. A. Wilson, \& F. C. Keil (Eds.), the MIT Encyclopedia of the Cognitive Sciences (MITECS) (pp. cxi-cxxxii). Cambridge, MA: MIT press.

Stalnaker, R. (2002). Common ground. Linguistics and Philosophy, 25(5/6), 701-721.

Tabach, M., Hershkowitz, R., Rasmussen, C., \& Dreyfus, T. (2014). Knowledge shifts and knowledge agents in the classroom. The Journal of Mathematical Behavior, 33, 192-208. https://doi.org/10.1016/j.jmathb. 2013.12.001.

Vygotsky, L. S. (1978). Mind in society: The development of higher psychological processes. Harvard University Press.

Vygotsky, L. S. (1986). Thought and language. MIT Press.

Werner, H. (1948). Comparative psychology of mental development. International Universities Press.

Werner, H., \& Kaplan, B. (1963). Symbol formation: An organismic-developmental approach to language and the expression of thought. Wiley. 
Yackel, E., \& Cobb, P. (1996). Sociomathematical norms, argumentation, and authonmy in mathematics. Journal for Research in Mathematics Education, 27(4), 458-477.

Publisher's Note Springer Nature remains neutral with regard to jurisdictional claims in published maps and institutional affiliations. 